\title{
Routine surveillance for bloodstream infections in a pediatric hematopoietic stem cell transplant cohort: Do patients benefit?
}

\author{
Heather Rigby $\mathrm{BSc}^{1}$, Conrad V Fernandez $\mathrm{MD}^{2}$, Joanne Langley $\mathrm{MD}^{3}$, \\ Tim Mailman $\mathrm{MD}^{3}$, Bruce Crooks $\mathrm{MD}^{2}$, Ann Higgins $\mathrm{RN}^{4}$
}

\begin{abstract}
H Rigby, CV Fernandez, J Langley, T Mailman, B Crooks, A Higgins. Routine surveillance for bloodstream infections in a pediatric hematopoietic stem cell transplant cohort: Do patients benefit? Can J Infect Dis Med Microbiol 2007;18(4):253-256.
\end{abstract}

BACKGROUND: Hematopoietic stem cell transplant (HSCT) recipients are at a high risk for late bloodstream infection (BSI). Controversy exists regarding the benefit of surveillance blood cultures in this immunosuppressed population. Despite the common use of this practice, the practical value is not well established in non-neutropenic children following HSCT.

METHODS: At the IWK Health Centre (Halifax, Nova Scotia), weekly surveillance blood cultures from central lines are drawn from children following HSCT until the line is removed. A retrospective chart review was performed to determine the utility and cost of this practice. Eligible participants were non-neutropenic HSCT recipients with central venous access lines. The cost of laboratory investigations, nursing time, hospital stay and interventions for positive surveillance cultures was calculated.

RESULTS: Forty-three HSCTs were performed in 41 children. Donors were allogenic in 33 cases (77\%) and autologous in 10 cases (23\%). There were 316 patient contacts for surveillance cultures (mean seven per patient) and 577 central line lumens sampled. Three of 43 patients (7\%) had clinically significant positive surveillance blood cultures. Bacteria isolated were Klebsiella pneumoniae $(\mathrm{n}=2)$ and Corynebacterium jeikeium $(\mathrm{n}=1)$. All follow-up cultures before initiation of antimicrobial therapy were sterile. All three patients were admitted for antimicrobial therapy if they were not already hospitalized and/or had an uncomplicated course. The estimated total cost of BSI surveillance and management of asymptomatic infection over six years was $\$ 27,989$.

CONCLUSION: The present study suggests that BSI surveillance in children following HSCT engraftment has a very low yield and significant cost. It is unclear whether it contributes to improved patient outcomes.

Key Words: Bloodstream infection; Hematopoietic stem cell transplant; Surveillance cultures

\section{La surveillance systématique des infections hématogènes dans une cohorte d'enfants ayant subi une transplantation de cellules souches hématopoiétiques : profitable aux patients?}

CONTEXTE : Les patients ayant subi une transplantation de cellules
souches hématopoïétiques (CSH) sont fortement prédisposés aux infections
hématogènes tardives (IHT). Toutefois, on ne s'entend pas sur les bienfaits de
l'hémoculture de surveillance chez les patients soumis à un traitement
immunodépresseur. Malgré l'usage répandu de l'examen, la valeur pratique de
la surveillance n'est pas clairement établie chez les enfants non neutropéniques
après une transplantation de CSH. MÉTHODE : Il est pratique courante d'effectuer, toutes les semaines, une hémoculture de surveillance chez les enfants ayant subi une transplantation de $\mathrm{CSH}$, à partir du cathéter central jusqu'au moment de son retrait, à l'IWK Health Centre, à Halifax, en Nouvelle-Écosse. Nous avons procédé à un examen rétrospectif de dossiers pour déterminer l'utilité et le coût de la pratique. Il s'agissait, dans tous les cas, de receveurs de CSH non neutropéniques, porteurs d'un cathéter veineux central. Nous avons tenu compte du coût des examens de laboratoire, du temps de soins, du séjour à l'hôpital et des interventions en cas d'hémoculture de surveillance positive. RÉSULTATS : Quarante-trois transplantations de CSH ont été pratiquées chez 41 enfants. Il s'agissait, dans 33 cas (77\%), de greffes homologues et, dans 10 cas ( $23 \%)$, de greffes autologues. Nous avons dénombré 316 contacts de patients pour les cultures de surveillance (moyenne de 7 par patient) et 577 prélèvements effectués dans la lumière des cathéters centraux. Des hémocultures de surveillance appréciables sur le plan clinique ont été décelées chez 3 des 43 patients (7\%). Les bactéries isolées étaient Klebsiella pneumoniae $(\mathrm{n}=2)$ et Corynebacterium jeikeium $(\mathrm{n}=1)$. Les hémocultures de suivi avant l'instauration du traitement antimicrobien étaient toutes négatives. Les trois patients ont été admis pour un traitement antimicrobien s'ils n'étaient pas déjà hospitalisés ou avaient connu une évolution sans complications. Le coût total estimé de la surveillance des IHT et du traitement des infections asymptomatiques sur une période de six ans s'élevait à 27989 \$.

CONCLUSION : La présente étude semble indiquer que la surveillance des IHT chez les enfants ayant subi une transplantation de CSH a un très faible rendement et coûte cher. Il subsiste toujours des doutes quant à son rôle dans l'amélioration des résultats.
Bloodstream infections (BSIs) and subsequent sepsis synBdromes are a frequent life-threatening complication of hematopoietic stem cell transplants (HSCT) (1-5). Patients are at a high risk for infection during the neutropenic period following HSCT and, despite neutrophil recovery, an increased risk for opportunistic infection persists for months or even years $(6,7)$. The presence of a central venous access device, which is standard of care in this population to secure vascular access for chemotherapy, transfusion support and nutrition, is a known additive risk factor for BSIs $(5,8)$. Strategies for prevention and pre-emptive intervention of BSIs include administration of prophylactic antibiotics and/or surveillance blood cultures. Evidence is mixed with respect to the efficacy of surveillance cultures. There is some evidence

${ }^{1}$ Dalhousie University; ${ }^{2}$ Department of Pediatric Hematology/Oncology; ${ }^{3}$ Department of Infectious Disease; ${ }^{4}$ Infection Prevention and Control Services, IWK Health Centre, Halifax, Nova Scotia

Correspondence: Dr Conrad V Fernandez, Department of Pediatrics and Bioethics, Dalhousie University, PO Box 9700, 5850/5980 University

Avenue, Halifax, Nova Scotia B3K 6R8. Telephone 902-470-7290, fax 902-470-7216, e-mail cufernan@dal.ca

Received for publication February 23, 2007. Accepted June 12, 2007 
that surveillance cultures may be warranted in HSCT patients during the neutropenic period before engraftment and for those on steroids $(2,5)$. However, surveillance cultures for BSIs in the absence of clinical symptoms are not recommended in the non-HSCT, immunocompetent populations (9-13).

BSIs in pediatric HSCT recipients occur at a lower frequency compared with adult populations, are associated with less morbidity and mortality, and are often associated with different pathogens (5,14-17). Most of the information about surveillance cultures in pediatric HSCT recipients is extrapolated from studies examining combined adult and pediatric populations. Therefore, the role of routine surveillance cultures remains uncertain.

The present study evaluates the utility of routine surveillance for BSIs in pediatric HSCT patients during the non-neutropenic period following HSCT until the central line is removed.

\section{METHODS}

The present study was approved by the IWK Health Centre (Halifax, Nova Scotia) research ethics review board.

\section{Study design and definitions}

The IWK Health Centre (IWK) is the only pediatric tertiary care medical centre in the Maritime provinces of Canada (population of two million). Eligible children are referred out of the region for HSCT and return to the IWK at a mean of 33 days post-transplant. They remain at the centre (primarily as outpatients) for a minimum of 100 days following HSCT. Surveillance blood cultures from all lumens of central venous lines are obtained weekly until the central line is withdrawn.

Data were obtained from three prospectively collected datasets for the period between 1999 and 2005. Eligible patients were identified through the pediatric hematology/oncology database. Information regarding insertion of central venous access devices and mechanical and infectious adverse events was obtained from the central venous access device database. Clinical information was supplemented from patient health records. Data were assembled for all children from the time of return to the IWK following HSCT until six months post-transplant or to the time of central line removal if less than six months. Data collection was deferred until neutrophil recovery if patients were neutropenic on return to the IWK.

The variables retrieved for the present study included sex, age, primary disease, donor source, type of central line, duration of neutropenia following HSCT, time to discharge from transplant centre, time to central line removal, graft versus host disease (GVHD) prophylaxis, antibiotic prophylaxis, number of surveillance cultures and number of lumens sampled. If a BSI occurred, information retrieved included the microbiology of infection, clinical management and course. Surveillance blood cultures were defined as all cultures from central lines taken from afebrile patients with no signs of local infection at the catheter site. Blood cultures taken within seven days of an unexplained febrile episode or following a positive blood culture were considered case finding and not surveillance cultures.

A clinically significant BSI was defined as a surveillance culture with a known pathogen, two consecutive sets of positive blood cultures with commensal flora from one or both lumens, or a positive culture from a patient with clinical signs of infection (18). The absolute neutrophil count was defined as the total of the neutrophil and the band form counts. Fever was defined as a temperature higher than $38.1^{\circ} \mathrm{C}$ over $4 \mathrm{~h}$ or a single temperature reading higher than $38.3^{\circ} \mathrm{C}$. Sepsis was defined as a positive blood culture in the presence of clinical signs such as fever. Neutropenia was defined as an absolute neutrophil count of less than $0.5 \times 10^{9} / \mathrm{L}$.

The cost of laboratory investigations, nursing time, hospital stay and interventions for patients with positive surveillance cultures was calculated based on the average nursing rate $(\$ 30 / \mathrm{h})$, estimated laboratory costs for negative cultures ( $\$ 13.22$ for one bottle and $\$ 18.62$ for two bottles), and estimated average cost for positive cultures ( $\$ 38.36$ per bottle). The cost of syringes was negligible with respect to the overall estimate and was, therefore, omitted from the calculations. The estimated average cost per inpatient hospital day was $\$ 865$. This estimate was provided by the hospital finance department specific to the average per-day hospital admission costs for the oncology unit between 2004 and 2005.

\section{Clinical management}

All patients had a surveillance blood culture obtained through the central venous line once weekly for most or all of the study period. Blood was drawn using sterile technique and the catheters were cleaned with chlorhexidine gluconate $0.5 \%$ and isopropyl alcohol 70\% (Steri-Stat, Matrix Medical Corp, USA) followed by isopropyl $70 \%$ rubbing compound. The volume of blood collected was determined based on the weight of the child. If the specimen was reported as positive, empirical antibiotics were commenced after a second blood culture was obtained.

All allogenic HSCT recipients received immunosuppressive therapy to prevent GVHD. The most common agents used were methotrexate and cyclosporine. Prophylactic penicillin was initiated in all patients at the time of engraftment and trimethoprim-sulfamethoxazole prophylaxis on neutrophil and platelet recovery. Both medications were continued for one year after transplant or two months after resolution of GVHD (whichever was last). Because this was a retrospective observational study, clinicians chose antimicrobial regimens for treatment of confirmed or suspected sepsis according to their discretion and empirical protocols at the institution.

\section{Demographics}

\section{RESULTS}

Over the study period, 43 HSCTs were performed in 41 patients, 21 of whom were male $(51 \%)$. The mean age was seven years (range one to 18 years). Thirty-three of 43 HSCTs were allogenic ( 15 were related [35\%] and 18 were unrelated [42\%]) and 10 of 43 HSCTs were autologous (23\%). The most common underlying illness leading to HSCT was leukemia in 19 of 41 patients (46\%), followed by solid tumour in seven patients (17\%), inborn errors of metabolism and Fanconi's anemia in four patients $(10 \%)$ and other illnesses in four patients (10\%).

Patients were hospitalized at the HSCT centre for a mean of 33 days (range four to 104 days) before being transferred back to the IWK; the mean time to engraftment was 16 days (range nine to 27 days; these data were missing from two patients). The mean length of study eligibility was 70 days (range seven to 176 days).

\section{Complications}

The incidence of sepsis during the period of surveillance cultures was zero. Of the 33 allogenic HSCT recipients, 16 developed GVHD (48\%), of which eight had acute GVHD alone, one had chronic GVHD alone and seven had both. One 
patient died of influenza A pneumonitis complicated by refractory hemolytic anemia.

\section{BSI surveillance}

Over the surveillance period, three of 43 blood cultures (7\%) met criteria for clinically significant BSI. There were 316 patient contacts for surveillance cultures (mean seven contacts per transplant) and 577 central line lumens were sampled. There were 105 patient contacts needed to detect one clinically significant positive surveillance culture.

The characteristics of the three patients with clinically significant BSIs are shown in Table 1. All three patients were afebrile at the time of the surveillance culture. Two of the cultures were drawn at regular outpatient follow-up appointments in patients who were clinically well. One was drawn from an inpatient initially admitted for GVHD but who had no clinical signs or symptoms of infection. Infectious work-up, including urinalysis, was negative in all three cases and the port sites were normal. In each case, the organism cultured was resistant to the given prophylactic antibiotics. All follow-up cultures performed before initiation of antimicrobial therapy were sterile. All patients were admitted for antimicrobial therapy if they were not already hospitalized for HSCT care. The total days in hospital directly related to treatment for the BSI for the three patients was 22 days (median five days). All three patients had an uncomplicated course.

The total estimated cost of BSI surveillance and treatment of asymptomatic infection over six years was $\$ 27,989$. This estimate includes the nursing time required to obtain the blood samples $(\$ 2,534)$, laboratory expenses $(\$ 5,555)$, medications administered for positive cultures $(\$ 870)$ and inpatient hospital stay $(\$ 19,030)$.

\section{DISCUSSION}

In the present study of a routine surveillance program for BSI in non-neutropenic pediatric recipients of HSCT, we found that asymptomatic BSI was an uncommon complication at this juncture of transplant. Furthermore, in cases where positive cultures were found, subsequent confirmatory cultures before initiation of antibiotics were negative. While our sample size was small, our results suggested that the effort and cost associated with this practice was of little benefit to this population. In addition, such a practice is not without harm. Accessing intravascular devices to obtain blood cultures may itself be a risk for infection, and adverse events may occur in relation to hospitalization for treatment of presumed BSI.

The efficacy of surveillance blood cultures has been evaluated in defined subsets of patients undergoing transplantation. In certain high-risk groups, there is evidence that surveillance cultures allow early detection of afebrile BSI before the development of sepsis. In a study of allogenic HSCT recipients, Chizuka et al (2) concluded that there may be a role for surveillance cultures in adult and pediatric patients on corticosteroids in whom the inflammatory reactions may be masked, and surveillance cultures may be the only diagnostic clue (2). Elishoov et al (5) examined adult and pediatric HSCT patients before discharge from the transplant centre and concluded that surveillance cultures may be warranted in the neutropenic period post-transplant. However, concern about occult BSIs may not apply to pediatric patients who are no longer neutropenic, are on antimicrobial prophylaxis and are clinically well. We suggest that surveillance should be
TABLE 1

Characteristics of patients with clinically significant surveillance blood cultures

\begin{tabular}{lccc}
\hline Characteristic & Patient 1 & Patient 2 & Patient 3 \\
\hline Pathogen & $\begin{array}{c}\text { Klebsiella } \\
\text { pneumoniae }\end{array}$ & $\begin{array}{c}\text { Klebsiella } \\
\text { pneumoniae }\end{array}$ & $\begin{array}{c}\text { Corynebacterium } \\
\text { jeikeium }\end{array}$ \\
Post-transplant, days & 30 & 32 & 42 \\
Post-CVAD insertion, days & 59 & 153 & 54 \\
Time to culture positivity, h & 10.7 & 8.5 & 29.0 \\
Age, years & 10 & 2 & 16 \\
Sex & Male & Female & Male \\
Donor & Related & Autologous & Unrelated \\
Immunosuppression at & Cyclosporine & None & Cyclosporine \\
the time of culture & & & No \\
Concurrent GVHD & Yes & No
\end{tabular}

CVAD Central venous access device; GVHD Graft versus host disease

targeted at higher risk patients in whom the positive predictive value is likely to be greater.

Evidence-based alternatives to surveillance cultures exist for the primary prevention of infection in both pediatric and adult HSCT recipients. Antibiotic prophylaxis (eg, penicillin) is effective in reducing infection rates from encapsulated bacteria (19). Trimethoprim-sulfamethoxazole is an effective chemoprophylaxis for pneumocystis pneumonia (Pneumocystis jiroveci) and may provide concurrent protection against other pathogens (20). In the first 100 days post-transplant, there is evidence in support of routine intravenous immunoglobulin for the prevention of bacterial infections in allogenic HSCT recipients with severe hypogammaglobulinemia (eg, immunoglobulin G less than $0.4 \mathrm{~g} / \mathrm{L}$ ) (19). Active immunization with pneumococcal and Haemophilus influenzae $B$ vaccines may be warranted after the first year post-transplant (21).

We found that the cost of performing surveillance blood cultures from hospital discharge to removal of the central line (or six months) for 43 HSCTs was $\$ 27,989$. This translates into a cost of $\$ 651$ per HSCT or $\$ 86$ per patient contact for surveillance culture. While a definitive conclusion cannot be made from a cost/benefit perspective, our cost estimate does highlight the need for further investigation into a significant health care expense.

In the present study, we have examined a small sample of pediatric HSCT recipients. A larger observational study in the pediatric age group would allow identification of high-risk groups so that surveillance cultures can be targeted. Furthermore, as a retrospective study, our conclusions are limited to patients who have been followed with the intervention. The need for more authoritative conclusions about the efficacy of surveillance blood cultures in pediatric HSCT recipients warrants a prospective clinical trial. However, our study has provided informative preliminary data for a topic that has not been extensively researched in HSCT patients.

The present study suggests that surveillance in children with central venous access devices who are afebrile and well has a very low yield and significant cost. Despite the use of this practice internationally, it is unclear that it contributes to improved patient outcomes.

ACKNOWLEDGEMENT: The present work originated out of the IWK Health Centre, Halifax, Nova Scotia. 


\section{REFERENCES}

1. Frère P, Hermanne JP, Debouge MH, de Mol P, Fillet G, Beguin Y. Bacteremia after hematopoietic stem cell transplantation: Incidence and predictive value of surveillance cultures. Bone Marrow Transplant 2004;33:745-9.

2. Chizuka A, Kami M, Kanda Y, et al. Value of surveillance blood culture for early diagnosis of occult bacteremia in patients on corticosteroid therapy following allogeneic hematopoietic stem cell transplantation. Bone Marrow Transplant 2005;35:577-82.

3. Czirók E, Prinz GY, Dénes R, Reményi P, Herendi A. Value of surveillance cultures in a bone marrow transplantation unit. J Med Microbiol 1997;46:785-91.

4. Krüger W, Rüssmann B, Kröger N, et al. Early infections in patients undergoing bone marrow or blood stem cell transplantation a 7 year single centre investigation of 409 cases. Bone Marrow Transplant 1999;23:589-97.

5. Elishoov H, Or R, Strauss N, Engelhard D. Nosocomial colonization, septicemia, and Hickman/Broviac catheter-related infections in bone marrow transplant recipients. A 5-year prospective study. Medicine (Baltimore) 1998;77:83-101.

6. Maury S, Mary JY, Rabian C, et al. Prolonged immune deficiency following allogeneic stem cell transplantation: Risk factors and complications in adult patients. Br J Haematol 2001;115:630-41.

7. Kalwak K, Gorczynska E, Toporski J, et al. Immune reconstitution after haematopoietic cell transplantation in children: Immunophenotype analysis with regard to factors affecting the speed of recovery. Br J Haematol 2002;118:74-89.

8. Kim DH, Bae NY, Sung WJ, et al. Hickman catheter site infections after allogeneic stem cell transplantation: A single-center experience. Transplant Proc 2004;36:1569-73.

9. Lidman C, Burman LG, Lagergren A, Ortqvist A. Limited value of routine microbiological diagnostics in patients hospitalized for community-acquired pneumonia. Scand J Infect Dis 2002;34:873-9.

10. Nielsen J, Kolmos HJ, Rosdahl VT. Poor value of surveillance cultures for prediction of septicaemia caused by coagulase-negative staphylococci in patients undergoing haemodialysis with central venous catheters. Scand J Infect Dis 1998;30:569-72.
11. Pillay T, Pillay DG, Hoosen AA, Adhikari M, Nowbath V. Utility of surveillance bacterial cultures in neonatal exchange blood transfusions. J Hosp Infect 1995;31:67-71.

12. Henke PK, Polk HC Jr. Efficacy of blood cultures in the critically ill surgical patient. Surgery 1996;120:752-8.

13. Levin PD, Hersch M, Rudensky B, Yinnon AM. Routine surveillance blood cultures: Their place in the management of critically ill patients. J Infect 1997;35:125-8.

14. Engelhard D, Elishoov H, Strauss N, et al. Nosocomial coagulase-negative staphylococcal infections in bone marrow transplantation recipients with central vein catheter. A 5-year prospective study. Transplantation 1996;61:430-4.

15. Engelhard D, Elishoov H, Or R, et al. Cytosine arabinoside as a major risk factor for Streptococcus viridans septicemia following bone marrow transplantation: A 5-year prospective study. Bone Marrow Transplant 1995;16:565-70.

16. Castagnola E, Garaventa A, Viscoli C, et al. Changing pattern of pathogens causing broviac catheter-related bacteraemias in children with cancer. J Hosp Infect 1995;29:129-33.

17. Uderzo C, D’Angelo P, Rizzari C, et al. Central venous catheter-related complications after bone marrow transplantation in children with hematological malignancies. Bone Marrow Transplant 1992;9:113-7.

18. Garner JS, Jarvis WR, Emori TG, Horan TC, Hughes JM. CDC definitions for nosocomial infections, 1988. Am J Infect Control 1988;16:128-40. (Erratum in 1988;16:177).

19. CDC, Infectious Disease Society of America, and the American Society of Blood and Marrow Transplantation. Guidelines for preventing opportunistic infections among hematopoietic stem cell transplant recipients. Recommendations of CDC, the Infectious Disease Society of America, and the American Society of Blood and Marrow Transplantation. Cytotherapy 2001;3:41-54.

20. Fishman JA. Prevention of infection caused by Pneumocystis carinii in transplant recipients. Clin Infect Dis 2001;33:1397-405.

21. Roy V, Ochs L, Weisdorf D. Late infections following allogeneic bone marrow transplantation: Suggested strategies for prophylaxis. Leuk Lymphoma 1997;26:1-15. 


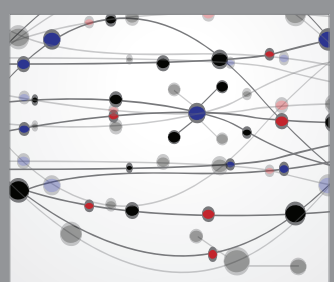

The Scientific World Journal
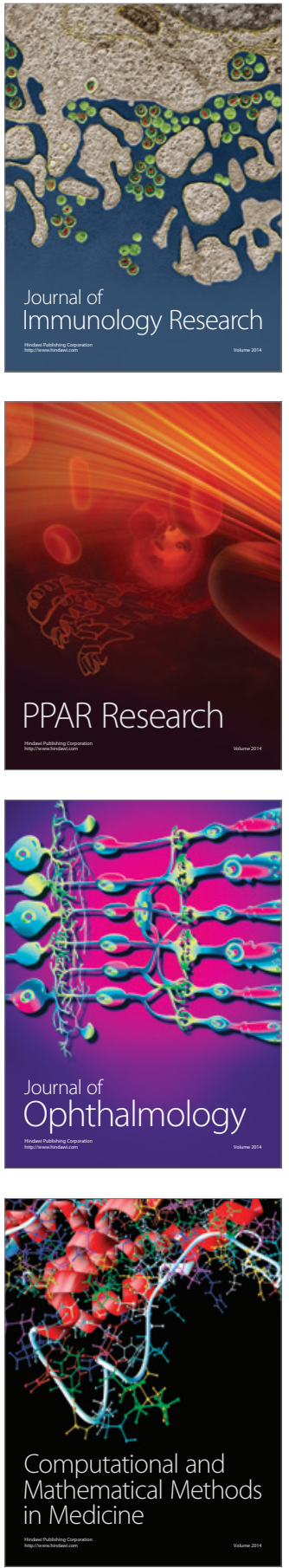

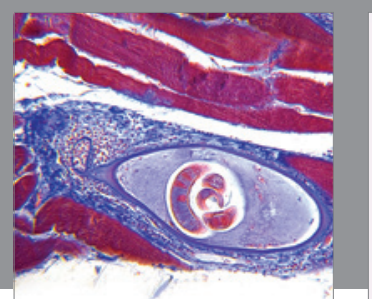

Gastroenterology Research and Practice

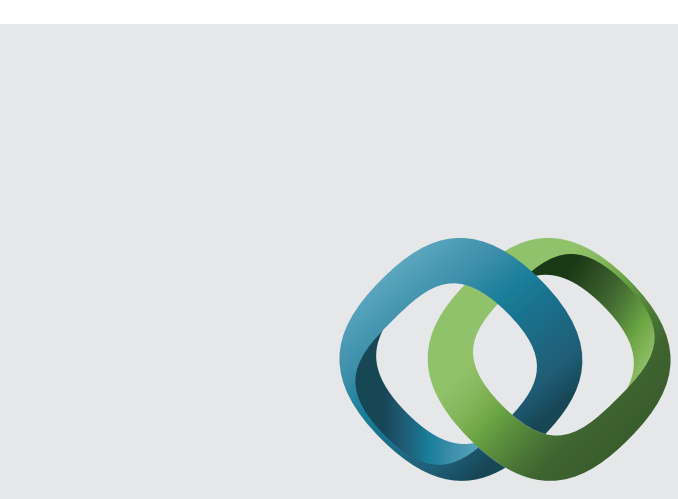

\section{Hindawi}

Submit your manuscripts at

http://www.hindawi.com
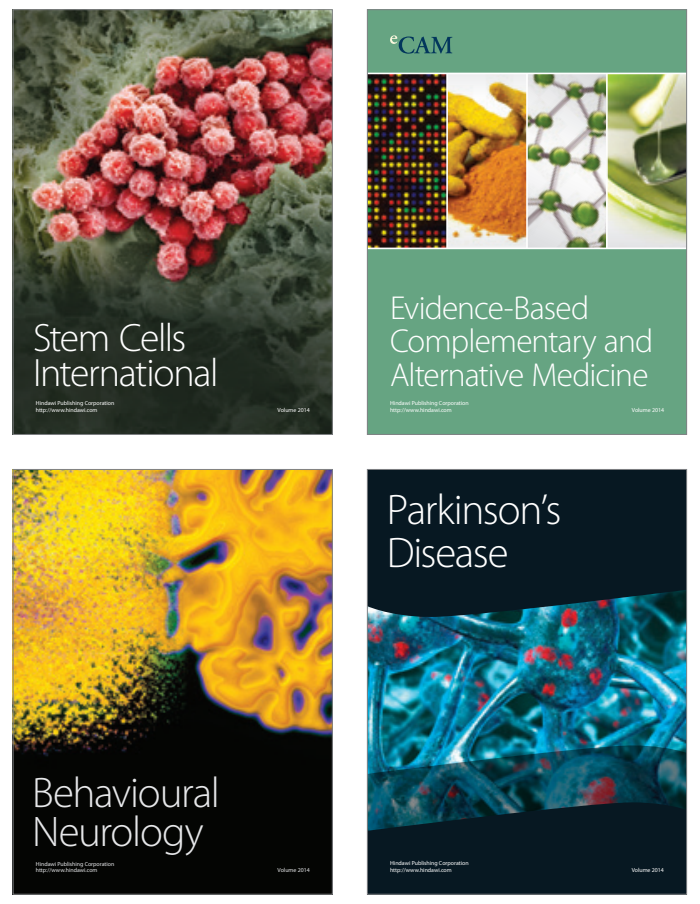
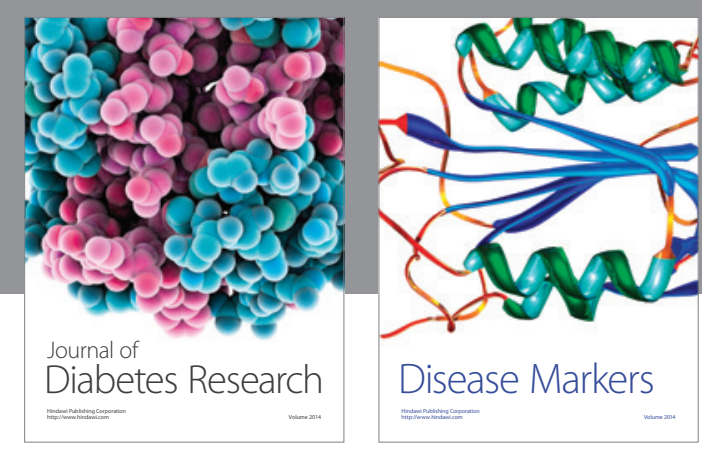

Disease Markers
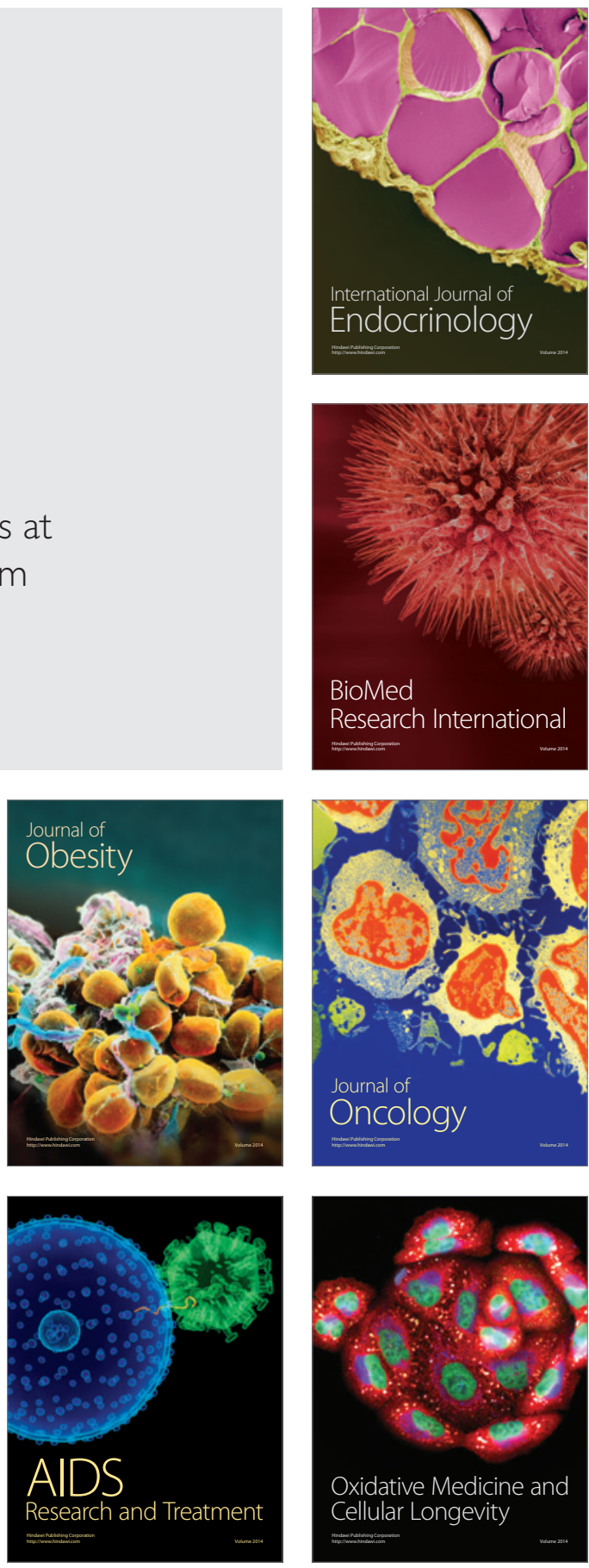